\title{
MULTI-SCALE MODELING OF TERRITORIAL DYNAMICS OF GEOSPATIAL ANTHROPOGENIC ENERGY CONSUMPTION
}

\author{
ANKUSH RAI*, JAGADEESH KANNAN R \\ School of Computing Science and Engineering, VIT University, Chennai, Tamil Nadu, India. Email: ankushressci@gmail.com
}

Received: 13 December 2016, Revised and Accepted: 07 April 2017

\begin{abstract}
Objective: The development of any region or territory stems from its own dynamic nature. Distribution and consumption of energy resources are varied territorially which in turn is ruled by the number of anthropogenic activities in association with geospatial localization. Such territorial dynamics necessitate considerable modifications of the energy infrastructure. Thus, the development of a computational multi-scale unified energy consumption model with the usage of geographic information helps in automating data analysis processes for sustainable urban planning, allocation of energy-saving infrastructures, and strategic deployment of the renewable energy resources to finely regulate the utilization of energy resources for sustainable energy consumption.
\end{abstract}

Method: The integration of city-wide energy system models and geographic information systems (GIS) is still in its infancy. The research work is divided into five interrelated work packages. The Modelling problem is achieved with the help of analytical hierarchical process. Thus, we propose a computational infrastructure for modelling city-wide geospatial energy consumption and automating the data analysis process to provide the sustainable environmental policy, which requires artificial intelligence-based geospatial aware comprehensive planning, regarding the modification of the energy supply, consumption, activities, and infrastructures in cities.

Results and Discussion: Thus, end result of the presented research work is fine-grained energy demand estimation from data sources, decentralized storage facility, and automated sustainable planning; investigation of GIS-based anthropogenic activities or mobility pattern influencing the wastage of energy resources, the transition from purely structural to operational planning, and, finally, the development of a new dynamic-based power market design.

Keywords: Computational modeling, Machine learning-based data analysis, Geographic information systems.

(C) 2017 The Authors. Published by Innovare Academic Sciences Pvt Ltd. This is an open access article under the CC BY license (http://creativecommons. org/licenses/by/4. 0/) DOI: http://dx.doi.org/10.22159/ajpcr.2017.v10s1.19744

\section{INTRODUCTION}

We are currently witnessing three essential trends with regard to energy infrastructure planning, increasing renewable energy generation and storage. [1-5] From planned production toward fluctuating production on the basis of renewable energy sources, from centralized generation toward decentralized generation, and from expensive energy carriers toward cost-free renewable energy carriers [6-12]. Technological solutions such as microgrids, smart cities, and smart buildings will only be successful when the urban planner knows where to concentrate energy resources and consequently estimate the potential for demand management, optimize the energy demands, install or share the energy resources [12-18]. Otherwise, the huge array of sensor networks deployed for smart energy management system for the reduction in energy consumption will ultimately be consuming more energy than what it is meant to do so [19-21]. Thus, the modifications of the energy infrastructure, necessitated by this increasing renewable energy use, require an extension of geospatial-based multi-scale modelling of anthropogenic energy consumption. Computational data analysis of such a model provides valuable input for sustainable policy generation to share/install energy infrastructure, precise settlement of energy storage infrastructures with respect to territorial dynamic [22].

Urban and territorial planning can be defined as a decision-making process aimed at realizing economic, social, cultural, and environmental goals through the development of spatial visions, strategies, and plans and the application of a set of policy principles, tools, institutional and participatory mechanisms, and regulatory procedures. Spatial planning covers a large spectrum of scales ranging from neighborhood, city/municipality, city/region/metropolis to national and supranational/transboundary and relies heavily on geographical information systems (GIS) to model the built environment [26-23]. It aims at facilitating and articulating decisions and actions that will transform the physical and social space and affect the distribution and flows of people, goods, and activities. Urban design is the multi-disciplinary process of shaping the physical setting for life in cities, towns, and villages; it involves the design of spaces, landscapes, building and group of buildings and the establishment of frameworks and processes that facilitate successful development. Thus, from the view of our research proposal, integrating GIS and energy system modelling enables the generation of a more complete picture of the overall energy system and future energy landscapes [24]. We claim that it is not enough to consider space and time as additional parameters, but in fact, space and time need to be fully integrated into energy system modeling processes to better understand the spatiotemporal dynamics of, for instance, energy demand, availability and the effectiveness of conventional and renewable resources, capacity and load patterns of energy infrastructures, including decentralized energy storages, and, finally, the return of investments and economic profitability.

Enhancing energy models with GIS data will significantly increase the granularity and complexity of these models. To deal with this, we propose innovative data reduction methods to extract a model of the (future) energy system that captures the interactions and relations that determine energy system efficiency, security, and sustainability.

\section{Related work}

In visions of a future smart energy and mobility system, smart grids match the supply from renewable energy sources with the demand from clean modes of transport such as electric vehicles. Consumers will play a large role in the transition to smart grids as their role changes from passive 
energy users to so-called prosumers and traders of flexibility in active demand-side management systems. The adoption and use of renewable energy technologies by these consumers determine the characteristics of these future smart energy systems. More specifically, the optimal configuration of a future smart energy system will greatly depend on the geospatial characteristics that influence both supply and demand.

The important role of geospatial characteristics in transition to a more sustainable energy system is widely recognized $[1,2,7]$. Various studies use GIS to support the planning process of renewable energy infrastructures. Specific applications include the classification of suitable spots for wind and solar farms, storage of hydroelectricity [1-3], and mapping of renewable energy resources, including solar photovoltaic (PV), wind, geothermal, biomass, and hydroelectricity [4-10]. These studies rely on geospatial data on land use, altitude, building structures, and infrastructure. Although the importance of demand-side factors in the transition to future smart and sustainable energy system is widely recognized, the vast majority of these studies address the potential energy supply without considering the demand side.

Including the demand side in models of the future smart energy system presents us with several challenges:

- The number and heterogeneity of demand-side factors provide a data challenge

- The number and heterogeneity of demand-side factors provide a modeling challenge.

In this research work, we address these two challenges by building innovative, data-driven computational models of the future energy system in two countries, India and The Netherlands. These cases are complementary as the Netherlands is characterized by high data availability, a stable, high level of electrification, whereas the Indian system offers abundance of data, but the supply of energy to massive population is a daunting task due to unavailability of energy distribution infrastructure. This is where the presented research work comes into play. The computational modeling will help the current Indian energy distribution, storage, and generation system more adaptive with the consumer's activities and enable an economic policy generation to establish energy reach in every home. A similar work computational framework based on ontology is achieved by Ramanathan et al. in 2004 and other works include the discovering patterns for human interaction system (2015).

Recognizing persistent patterns in energy consumption will improve energy planning and correct dimensioning of the energy infrastructure. In urban areas, patterns exist in the energy consumption in various types of buildings. In this research work, we will identify energy consumption patterns in the cities using fine-grained big data. Estimation of building energy consumption has been a field of study for a long time (Zhao and Magoules 2012). Most studies distinguish between residential and commercial buildings. For residential buildings, it has been shown that inhabitant's behavior is most important for estimating energy consumption (Pettersen, 1994). For commercial buildings, behavior is generally more predictable.

In this research, we will develop a prediction model based on the following data: (1) Physical characteristics of buildings such as dimension in square meters and European Energy Label, (2) occupant information such as behavioral patterns learned from data and working schedules in commercial buildings, (3) geographical information such as building's address, postcode, and geographic orientation, (4) weather meters including temperature, humidity, wind, and duration of sunshine, and (5) gas and electricity consumption. The data from the model are used to both inform spatial planning and design data-driven business models for smart energy systems. Profitable business models for value creation and value capture in smart energy systems are pivotal to realize the transition to smart and sustainable electricity grids. In addition to knowledge regarding the technical characteristics of smart grids, we need to know what drives companies and consumers to sell and purchase services in a smart grid. In a recent review of business models for smart grid services in 434 European and US smart grid pilot research works, Niesten and Alkemade (2016) found that pilots most often discuss three types of smart grid services: Vehicle-to-grid and grid-to-vehicle services, demand response services, and services to integrate renewable energy. Knowledge regarding demand response services is restricted to different types of value creation and capture. The review indicated that business models can be profitable when a new actor in the electricity industry, that is, the aggregator, can collect sufficiently large amounts of load.

Such an analysis requires more data and more advanced analysis methods in association with dynamic geographical variables, for example, climate conditions. Hence, further research in this direction will serve as the premise for a more extensive framework that fuses GIS, mathematical optimization, and simulation so as to locate the optimal area optimal size for the suitability of location for installation of PV solar plants aimed at grounds environments. This will help in decisionmaking process for locating specific geographical sites that will soothe the requirements of installation of renewable energy reservoirs where the climate conditions are more susceptible to match the requirements or contribute continuous drive power for the generation of energy, for example, selection of geographical location for the installation of windmill based on climate conditions where their incessant wind flow is ensured. Likewise, this approach will coordinate toward the specific localization of extra spatially differing renewable sources of energy, for example, solar, wind, hydro, bio, or geothermal energy.

Establishment of sustainable energy network and planning is impacted by different dynamic variables. In locales where the potential for expanding territorial energy supplying networks wanders in various regions, the monetary expenses of energy creation, transmission, and circulation are hard to estimate. Subsequently, considering the geographic segment, utilizing geospatial methods and GIS is a basic part in finding the limits to which such an extension is economically attainable [11]. In spite of the fact that, the most effective utilization of energy storage innovation, for this situation, energy storages, likewise, relies on upon spatial parameters, for example, the separation to the following piece or to individual houses. The optimal utilization of energy storages in energy generation, transmission, and dissemination might altogether impact the general expenses of supply ranges. Subsequently, energy supply decisions ought to be founded on the spatial arrangement of the energy demands and the qualities of the nearby energy supply unit area, which verifiably supports the necessary need of GIS. In addition, the thought of geospatial perspectives in local energy system optimization is especially significant for accurately deciding the supply region if the emphasis is on grid-associated technological advances [12]. It is inferred from such studies that, among geospatial parameters, the topological parameters, for example, availability or nearness of a gridassociated energy infrastructure, constitute crucial data for both viable planning and agent errands.

Aydin et al. present a methodology in view of GIS, fuzzy set theory, and multi-criteria decision-making for finding the optimal arrangement of a half-breed wind-solar-PV renewable energy system, which can possibly diminish the requirement for energy storage [1]. Their primary contention is that, contingent upon climate and atmosphere conditions, one renewable energy source is supplementing the other. As it was, shortcomings and qualities of the individual systems adjust for each other. Notwithstanding, this methodology might just be material if enough space for power plants is accessible, which is not really the case, for occasion, in thickly developed urban environments. An extra restricting variable is that building proprietors can choose exclusively if and how to utilize renewable energy. In this manner, the individual utilization of renewable energy sources is as a rule foreordained in that conceivable areas for setting up renewable energy power plants are uncommon in thick urban ranges. Similarly, Omitaomu et al. portray an adjusted GIS-based multi-criteria decision analysis way to deal with decide the suitability for new power creating destinations [13]. The methodology considers ecological, 
geographical, and financial perspectives, among others. Despite the fact that this GIS-based methodology is intended to work everywhere scales, it is spatially express in that it isolates the whole zone of the USA into a huge number of $100 \mathrm{~m} \times 100 \mathrm{~m}$ cells and registers the suitability of every cell for new power generation locales. Huge downsides of methodologies such as mentioned in $[1,13]$ incorporates the absence of capacity to consider more dynamic spatiotemporal perspectives crosswise over various spatial and temporal scales, or the lacking integrability of topological parts of the basic energy network as respects the adjusting of uncommon features in energy load shifts crosswise over spatially distributed energy storage cells.

The authors of Strachan et al. [14] present an illustration of such a methodology, to the point that is secured inside of economically inclusive energy systems model of the U.K. For the German hydrogen economy, Ball et al. present an optimization approach for getting to the geographic and temporal parts of a hydrogen transport infrastructure design [15]. The results of both studies uncover that the utilization of GIS is pivotal while investigating the effect of the geospatial measurement of hydrogen networks and the expanding changes in energy generation blend on future energy system infrastructures and supply chains. The presented venture gives a flexible modeling stage that will empower organizers, earthy people, and governments to ascertain, gauge, and screen the ecological effect of changes in the energy blend on nearby, territorial, and global scales. The venture is a careful way to deal with incorporating GIS and energy system models, with clear and exploitable results. Coupling GIS with energy system modeling is likewise included in the field of hydrogen supply, demands, and infrastructures.

\section{METHODS}

The research work is divided into five interrelated work packages. The Modeling problems that we address in our computational research work are summarized as follows:

Problem set I: Geospatial awareness for energy consumption in integration of anthropogenic energy consumption actions.

Problem set II: Integration of networked energy transmission topologies into GIS for geo-location-based energy demand estimation.

Problem set III: Policy generation for planning and estimation of decentralized renewable energy installation/sharing/storage facilities for sustainable energy consumption.

Problem set IV: Dynamic modeling for power market design.

One of the key issues that we strive to overcome in this research work is to reduce the complexity of GIS and energy system models. We propose to do so using techniques from machine learning. By learning models for patterns in the interactions between the many parameters of these two models, we can discover which combinations of parameter values can and cannot occur in practice. By limiting the parameters to take on values that are consistent with a predictive model's expectations, we greatly reduce the number of possible assignments to these parameters. In recent work (Verwer et al., 2015), we have demonstrated that models learned from data can be efficiently combined with mathematical models in a white-box fashion, meaning that the entire model is translated into mathematical formulations that can be used inside the reasoning engine of a planning system. Such a generic approach is very powerful since it allows the use of sophisticated methods for mathematical simulation and optimization. In particular, we will investigate the use of modern model order reduction methods in combination with these learned models. Model order reduction (Schilders et al., 2008) is a mathematical method that aims to simplify or approximate mathematical formulations, reducing the number of parameters, to make simulation and optimization computationally feasible. Intuitively, the learned models impose tight constraints on the possible parameter values. A model order reduction technique can use these constraints to remove parameters that can be directly determined from others. Since these relations are learned from data, these constraints do not need to hold in general. We only care about whether they hold in practice.

Geo-specific modeling involves the search for the best location of one or more facilities to support some desired function. The current impediments to the application of location models, issues associated with the integration of location models into GIS, and future needs in GIS functionality to support location models are to come up a modeling technique with which the passing of weighted features, decision sets, and cascaded fitting examination of multiple coexisting conditions could be addressed with the same method. This is where the presented dynamic Boolean network (DBN) stands out as it not only supports examination of multiple conditions simultaneously but also addresses the hierarchical evolution of weighted feature vectors from one consequent stage to another.

Each of the above problems has hierarchical association, for which achieving a comprehensive solution for data-driven sustainable energy modeling requires an approach to pass on the filtered processed decision formulated from the processing of Problem set I to Problem set II and so forth. Problem set I involve the searching for an appropriate location for an activity. This problem can be called the locational search problem. It is common to refer to the locational search problem as a facility location problem. This type of problem can involve the placement of one activity or the placement of a set of interrelated facilities. Such problems are called single- and multi-facility location problems. For example, the weighted feature vectors and decisions sets regarding suitable sites with reduced anthropogenic energy consumption action are to be forwarded for modeling and processing the data for Problem set II. The same weighted feature vectors and decisions sets are usable in association with network energy transmission topologies to derive the results whether the location and transmission of energy sources are enough to supply the geo-specific energy needs or what is the energy demand of the location in particular period of time or even where energy can be conserved at specific point of time. Such that the weighted patterns extracted from modeling of previous step is forwarded for proper computational planning or policy generation for the facilitation of installation/shared/storage of renewable energy resources or its suitability with particular region at particular period of time. Finally, the decisions sets and feature vectors so extracted from modeling of Problem Set IV shall be used for including flexible demand, energy service providers, and renewables to model the dynamic nature of the evolving power market. In the end, this will help users in specific location to cut short the wastage of power supply. For example: If the climate condition with respected to specific geo-location suggests that humidity shall be high in evening, then the user shall be liable to save power in morning time by switching off air conditioners and utilizing it when it is certainly required. This is where smart grids, smart cities, smart buildings and internet of things (IoT) come into play. The presented research provides a computational infrastructure for such sensor-based infrastructures (smart grids, smart cities, smart buildings and IoT) by intelligently processing and understanding the territorial dynamics of the energy resources. Later, the decisions sets or policies are to be forwarded to smart infrastructures. Otherwise, simply flooding the energy distribution network with array of sensors will only lead to more extensive usage of energy supply than what it is meant to reduce.

The modeling approach can be summarized as: Suppose that we divide a city into approximately 60000 cells, each measuring 78 acres. In each cell, 62 variables were measured, including land use, land cover, proximity to a water source, and seismicity. Each of these variables was converted to a subscore function and added together to form a site suitability score. Single-site location analysis can often be approached with some form of ranking or scoring process, which is based upon a set of attributes. From this, a map was produced which identified those cells which scored higher than one standard deviation above the mean. Information associated with various attributes is typically converted to subscores and combined into suitability scores for compatibility with use for the next problem set. Next, a network is defined where the nodes represent the centroids of the cells in the form of arcs pointing from state $\mathrm{x}$ to state $\mathrm{y}$ with time dependency as one of the dimension. The arcs depict possible directions 
which can be taken by the problem set through a given cell. Thus, chances are there for considerable error, which may exist in the networking of features based upon the certain attribute. This can be eliminated with the co-simulation model of DBN. The decision sets extracted by solving on problem will act as an attribute for that cell, and hence, the costs or weights can be defined as some weighted combination of the suitability scores through which the network passes. A case is depicted where the problem set has a width of influence upon the weights and is associated with the footprint of the next problem set in a given cell. Given a topological network, complete with arcs and associated costs of operation, feasibility of a decision can be identified as the least cost (most suitable) outcome. The beauty of this DBN process is that it can be solved optimally for large problems where the processing time for the desirable outcome is few hours on modest workstations. Because this process is fast, it can be repeated for a wide variety of parameter, weights associated with different components of the suitability score, allowing us to test the sensitivity of the variation to various levels of importance weights. The more efficient solution from cosimulation supported by DBN algorithm can be made for a given application, the more flexibility there is in order to check for fitness of data and sensitivity analysis.

We now consider the new compression scheme in more detail. It is based on an atypical form of vector clustering, which merges groups of deterministic test cubes using the logical formulations of autonomic hierarchy process while allowing some degree of their incompatibility. The overview of the method is shown in Fig. 1. Here, the test data are compressed into scan chains based on the following logic. Let $C_{k}$ be the test vectors where $\mathrm{k}=1,2,3, \ldots \mathrm{n}$ of size $\mathrm{m} \times \mathrm{n}$ and $\mathrm{n}$ is the block size of data. The data need to be pre-processed or divided into scan lines of test vectors as follows:

$$
C_{k}=\sum_{i=1}^{m} C_{0}\left(K_{i}\right)+\sum_{i=1}^{K_{1}} C_{0}\left(b_{i}\right)+\ldots+\sum_{i=K_{m-1}+1}^{K} C_{m}\left(b_{i}\right)
$$

where $C_{i}($.$) is the required size to encode the indices value at each$ section. Here, the partitioned scan lines are decomposed with the help of combined approach between autonomic hierarchy process to perform pair-wise comparison judgments. However, to achieve better parameterization, we have used fuzzy logic.

Here, the decomposition principle calls for structuring the hierarchy to capture the basic elements of the problem. This step in the context is to handle a variety of complex and comprehensive multi-criteria decisionmaking problems which have several alternatives to implement and quite a lot of criteria are used to check and evaluate the implementation. To apply the fuzzified analytic hierarchy process (FAHP), initially, the comprehensive problem needs to be structured into different hierarchical levels with regard to the properties or attributes of the problem considered. Now, for each fuzzy set $X$, the membership function is given by $\mu_{A}$ which takes the value of the interval $[0,1]$, i.e., $m_{A}: X \rightarrow[0,1]$. Thus, the fuzzified membership values can be represented as:

$$
X=\frac{m_{A}\left(n_{1}\right)}{n_{1}}+\frac{m_{A}\left(n_{2}\right)}{n_{2}}+\ldots+\frac{m_{A}\left(n_{p}\right)}{n_{n}}
$$

where the trapezoidal membership function for $\mathrm{m}_{\mathrm{A}}$ is given as:

$$
m(n)\left\{\begin{array}{cc}
0, & x \in[-\infty, l] \\
\frac{n \quad l}{n \quad m}, & x \in[l, m] \\
1 & x \in\left[m_{1}, m_{2}\right] \\
\frac{u \quad n}{u \quad m}, & x \in[m, u] \\
0, & x \in[u,+\infty]
\end{array}\right.
$$

$m_{A}(n)$

Here, $\mathrm{m}_{1}$ and $\mathrm{m}_{2}$ are the two membership functions, followed by $\mathrm{l}$ and $\mathrm{u}$ are the respective upper and lower limits where the sub-membership functions are bounded within $l \leq m_{1} \leq m_{2} \leq \mu$.

Since we have already established why our own newly mathematically modeled coactivated Boolean networks for model formation through machine learning algorithm is the best solution, now we discuss the mechanism of DBN (Fig. 1a). Unlike current neural network, the DBN is modeled on much more deeper level (Fig. 1b). The biological neuron consists of several networks of microtubules which enable each neuron to response based on the data type or make it data driven (Fig. 1c). Thus, the current DBN which we are inclined to use will enable the computational framework to model the feature sets by enabling the check for conditional existence of other multiple-dependent conditions

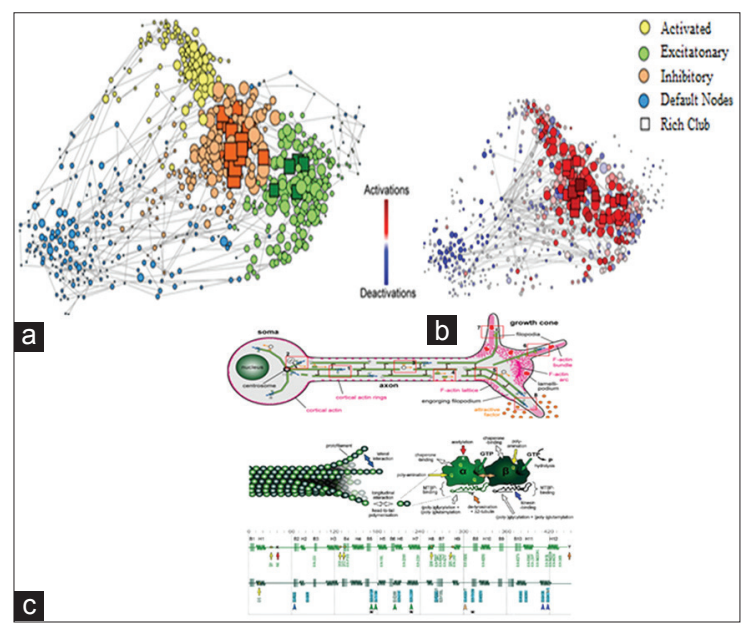

Fig. 1. Topological representation of the functional coactivation network. (a) Force-based layout of the minimum spanning tree is used to locate nodes in relation to their topological (rather than anatomical) proximity to each other. Different modules are coded by color, and rich club nodes are represented by squares, with the size of all nodes proportional to their weighted degree (strength). (b) Nodes in anatomical space, colored according to proportion of times they present activations and deactivations. (c) Illustration of deeper microtubule connection within a biological neuron which is computationally modeled in DBN to have better learning efficiency with plasticity and elasticity in DBN with better chances of forming correlation with the given data and respond intelligently in data-driven scenario, nodes arranged in the same layout as a, and colored as in $\mathbf{b}$. In addition, note that the rich cluster concentrates most of the activations, whereas the periphery and particularly the default-mode network concentrate the deactivations (Fig. 1). Edges represent the top 1 percentile of most consistently reported activation and deactivations (no directions shown for clarity purposes). Edges can be seen spanning across different modules. Although the network cost is usually found to be overall low, as measured by the distance of connections, the network topology still managed to balance integration and segregation between all topological artificial neural regions: The clustering of the network threshold at sparse levels is much higher than random, while retaining a similar path length. In all these respects, the organization of the coactivation network is convergent with properties of a comparable functional connectivity network generated from resting-state of excitatory sequences. As known from prior study, and reproduced here, a pattern recognition state of polymorph neural networks for feature extraction and encoding of it for both the unstructured data and semantic datasets (which is an example of small world encoding), with fat-tailed degree distributions and parsimonious distance distributions 


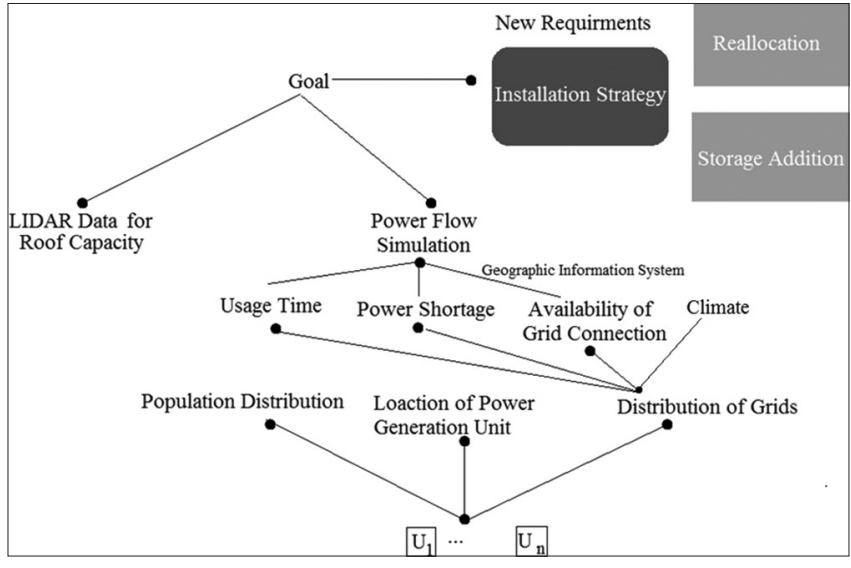

Fig. 2: Illustration of fuzzified analytic hierarchy PROCESS for the preliminary study. Note that only static population and climate data ae taken into account in this study

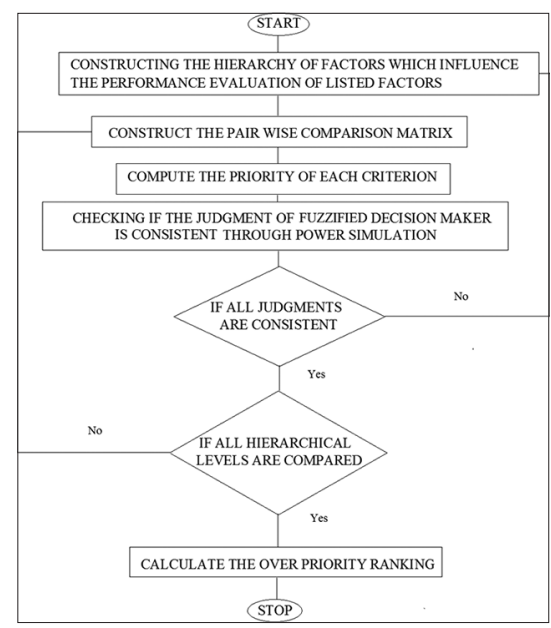

Fig. 3: Flowchart of the control flow processing of fuzzified analytic hierarchy process

called coactivation [25-27]. This coactivation network is employed for the computation as it is topologically complex in several ways and we intend to use it on our research with the combination of evolving Boolean networks. The nodal degree distribution was fat-tailed with high-degree hub nodes to be located in presented polymorph neural network using sequence of information to excite the necessary regions and assess the information in an associative form. This enables the machine not only to learn but also embark the cross relationship between various data for prediction or simulation-based logical conclusion. Computationally, this topology was embedded parsimoniously, in terms of the connection distance between coactivated nodes. Most connections or edges were separated by short sequence of excitatory data, significantly shorter than random networks; with $\mathrm{p}<10^{-3}$ in the permutation test. Relatively few edges were long distance, and these were often interhemispheric research workions between bilaterally homotopic regions where $14 \%$ of longest connections (defined as top 10 percentile) were homotopic; significantly more than random.

This allows the wiring of the learned neurons to be plastic (left, which rigidly affinity to particular sequence of data and exhibits the constant state once excited) and the elastic neurons (right, which returns to its initial state; mainly used for transfer of sequence from one network point to other). The spot and arrow above each receptor represent excitation that stimulates the pixel receptor. The sequence of numerical response units which is generated by the elastic and plastic neurons helps create responses to spot intensity of 2.0. For the coactivation network, it is possible to assign functional as well as anatomical labels to the modules using Boolean networks $[28,29]$. To do this, we will consider the high-level behavioral domains used which describe each contrast in the primary literature: Geospatial specificity, anthropogenic action-interaction, perception, and interception. We then labeled each edge according to the domain most frequently causing coactivation of the corresponding pair of regions. In the occipital module, the highest proportion of intramodular modeling is set aside, as once modeled, the polymorph neural network has proven its evolutionary nature and self-modeling in dynamic scenario in previous studies corresponded to coactivation by perception (39\%) and the other domains coactivated $<20 \%$ each; similarly, in the default-mode module, other domains each accounted for $<21 \%$; whereas, in the central module, $62 \%$ of intramodular edges were coactivated by action. Thus, it seems reasonable to say that the presented research has firm prominence with its scope fulfillment and is relatively specialized for action, the occipital module for vision-based perception. Action and cognition tasks in regard to weather prediction accounted for approximately the same proportion of intramodular edges in the other software AI modules (34\% and 38\%, respectively), and therefore, we described it as specialized for such modeling of executive functions.

Geo-specific modeling involves the search for the best location of one or more facilities to support some desired function. The current impediments to the application of location models, issues associated with the integration of location models into GIS, and future needs in GIS functionality to support location models are to come up with a modeling technique with which the passing of weighted features, decision sets, and cascaded fitting examination of multiple coexisting conditions could be addressed with the same method. The computational framework is divided into three phases.

Phase I of this research included creating knowledge acquisition procedures to produce a group of data that could be helpful in cascading the relationship between the factors, for example, geographical topologies, energy usages, populace, and climate connected with each other; we will utilize FAHP. Analytical hierarchy process alludes to a decision strategy used to rank order of an arrangement of options in light of different criteria. Finally, analytic hierarchy process has been broadly used as a multiple criteria decision-making tool or a weight estimation technology in many research fields, including determination, evaluation, planning and improvement, decisionmaking, and forecasting. The traditional analytic hierarchy process algorithm needs to correct judgments. Especially, considering the unpredictability and vulnerability required in decision issues, it is once in a while improbable or even difficult to require precise judgments. Subsequently, it is more regular or reasonable that a decision-maker is permitted to give fuzzified judgments. The learning acquisition in Phase I delivered a modular methodology for the evaluation of the membership functions of relative combinatoric factors impacting the system (Fig. 2).

Phase II includes usage of results from Phase I for network generation. For the uniform network, all individuals test units have the same degree. We utilize the configuration model, communicated with a stub connection algorithm, to make arbitrary graphs with a predefined degree dissemination. By haphazardly associating individual sets, we diminish higher order structure. For every node, we first allot it a degree $\mathrm{k}$, and after that, we make a set of $\mathrm{k}$ stubs that represent each of these edges with just a solitary tail association with a node. We rehash this for all nodes and after that join these stubs into an expert set. This set is then arbitrarily separated into equal parts, and a stub from every subset is coordinated to one from the other subset, shaping a complete edge [30]. On the off chance that there is an uneven number of stubs, an arbitrary individual set is given an additional stub. We do not permit self-connections or copy edges between nodes (Fig. 3).

For the gamma-distributed-degree organize, every individual is doled out a degree drawn from a discretized rendition of the gamma 
distribution with a mean degree. The gamma dissemination was chosen because it permits the mean and fluctuation to be changed freely, with any difference somewhere around zero and limitlessness conceivable. Discretization was performed by first drawing an arbitrary number from a persistent gamma distribution with mean qualities, adjusting to the closest whole number, and after that including one. It was affirmed numerically this made a circulation with the coveted properties over the required scope of mean and standard deviation values. The system is then made utilizing the stub interface algorithm. An edge is built between every pair of collective factors with a likelihood $p$, free of the presence of different edges. The subsequent degree dispersion is binomial. Every edge of each node is then rewired with likelihood p. Rewiring includes separating from the distal node and associating with another arbitrary non-self and non-neighbor node such that double edges are avoided and the uniform degree of the system is preserved.

Phase III: Now, for the prediction of the energy usage data, the branching process calculations of the network so formed in Phase II can tell us about the probability of usage emergence by capturing stochastic effects that are important when analytic hierarchy process levels are subside with one another but do not accurately capture the dynamics as prevalence levels become significant. For this task, which gives forecast on deterministic models that track both live data and susceptible network nodes are appropriate. This is where the presented coactivated DBN stands out as it not only supports examination of multiple conditions simultaneously but also addresses the hierarchical evolution of weighted feature vectors from one consequent stage to another [31]. Each of the above problems has hierarchical association, for which achieving a comprehensive solution for data-driven sustainable energy modeling requires an approach to pass on the filtered processed decision formulated from the processing of hierarchical levels to consequent hierarchical levels and so forth.

\section{RESULTS AND CONCLUSION}

There are many common challenges in multiscale modeling, including validation and design of tools for programming and executing multiscale simulations. This presented theme issue seeks to establish common frameworks for theoretical modeling, computing, and validation and to help practical applications to benefit from the modeling results.

- It is observed that multiscale problems do not typically have a closed solution (except for some idealized situations when a single-scale model at the finest level). To simulate a large enough system with multiple scales at the level of detail required, one has to combine models at various scale resolutions and invariably deal with different physics. Multiscale systems can be characterized by the fact that there is a form of approximation or coarse graining involved in the multiscale modeling, corresponding to an error below some threshold scale of interest.

- On the other hand, it is not possible to coarse grain everything as it incurs a loss of information at each step. Coarse graining also involves the exchange of information between the fine scale and the coarse scale. In some cases, this can be approximated as a one-way coupling between the scales, but, in others, a fully two-way coupling framework is required.

- The exchange of information between multiple scales leads to error propagation within the multiscale model, thus affecting the stability and accuracy of the solution. Furthermore, it probes the question as to whether any mutual approaches for careful error analysis can be carried out at a theoretical level.

- Without thorough analysis or DBN-based guidance for computational modeling, it is necessary to make a comparison by empirical validation, or with a high-fidelity single-scale model, if that is computationally tractable. In numerous multiscale systems, a sequential approach is adopted when building a hierarchy of models. These begin with a high-fidelity model at a single-scale well established with regard to the experiment or observation, which sequentially transfers information to a more coarse-grained level.
The provided solution for a multiscale model in energy setting is a hybrid method for bridging continuum of energy usage and other factors influencing it. Apart from mathematical complexity and software optimization at the conceptual level, another issue presented is how to implement multiscale models in practice at the computational level. For example, there is the issue of coupling different codes written for single-scale single physics simulation in a unified framework. It is necessary for the latter to be flexible enough to accommodate new codes written in an object-oriented environment in addition to legacy ones used in different communities for many years and based on more traditional data structures.

\section{REFERENCES}

1. Aydin NY, Kentel E, Duzgun HS. GIS-based site selection methodology for hybrid renewable energy systems: A case study from Western Turkey. Energy Convers Manag 2013;70:90-106.

2. Janke JR. Multicriteria GIS modeling of wind and solar farms in Colorado. Renew Energy 2010;35:2228-34.

3. Connolly D, MacLaughlin S, Leahy M. Development of a computer program to locate potential sites for pumped hydroelectric energy storage. Energy 2010;35:375-81.

4. Van Hoesen J, Letendre S. Evaluating potential renewable energy resources in poultney, vermont: A GIS-based approach to supporting rural community energy planning. Renew Energy 2010;35:2114-22.

5. Ramachandra TV, Shruthi BV. Spatial mapping of renewable energy potential. Renew Sustain Energy Rev 2007;11:1460-80.

6. Zambelli P, Lora C, Spinelli R, Tattoni C, Vitti A, Zatelli P, et al. A GIS decision support system for regional forest management to assess biomass availability for renewable energy production. Environ Model Softw 2012;38:203-13

7. Kaundinya DP, Balachandra P, Ravindranath NH, Ashok V. A GIS (Geographical information system)-based spatial data mining approach for optimal location and capacity planning of distributed biomass power generation facilities: A case study of Tumkur District, India. Energy 2013;52:77-88.

8. Zhang F, Johnson DM, Sutherland JW. A GIS-based method for identifying the optimal location for a facility to convert forest biomass to biofuel. Biomass Bioenergy 2011;35:3951-61.

9. Sultana A, Kumar A. Optimal siting and size of bioenergy facilities using geographic information system. Appl Energy 2012;94:192-201.

10. Eising J, van Onna T, Alkemade F. Towards smart grids: Identifying the risks that arise from the integration of energy and transport supply chains. Appl Energy 2014;123:448-55.

11. Nielsen S, Möller B. GIS based analysis of future district heating potential in Denmark. Energy 2013;57:458-68.

12. Biberacher M, Hausl S. Räumliche Aspekte in der Regionalen Energiesystemoptimierung. In: Strobl J, Blaschke T, Griesebner G, editors. Angewandte Geoinformatik AGIT 2012. Berlin, Germany: Wichmann Verlag; 2012. p. 290-304.

13. Omitaomu OA, Blevins BR, Jochem WC, Mays GT, Belles R, Hadley SW, et al. Adapting a GIS-based multicriteria decision analysis approach for evaluating new power generating sites. Appl Energy 2012;96:292-301.

14. Strachan N, Baltaozkan N, Joffe D, McGeevor K, Hughes N. Softlinking energy systems and GIS models to investigate spatial hydrogen infrastructure development in a low-carbon UK energy system. Int J Hydrogen Energy 2009;34:642-57.

15. Ball M, Wietschel M, Rentz O. Integration of a hydrogen economy into the German energy system: An optimising modelling approach. Int J Hydrogen Energy 2007;32:1355-68.

16. Haas R, Auer H, Biermayr P. The impact of consumer behavior on residential energy demand for space heating. Energy Build 1998;27(2):195-205.

17. Pettersen T. Variation of energy consumption in dwellings due to climate, building and inhabitants. Energy Build 1994;21(3):209-18.

18. Schilders WH, van der Vorst HA, Rommes J, editors. Model Order Reduction: Theory, Research Aspects and Applications. Vol. 13. Berlin, Germany: Springer; 2008.

19. Zhao H, Magoules F. A review on the prediction of building energy consumption. Renew Sustain Energy Rev 2012;16(6):3586-92.

20. Verwer S, Zhang Y, Ye QC. Auction Optimization using Regression Trees and Linear Models as Integer Programs, Artificial Intelligence. DOI: 10.1016/j.artint.2015.05.004.

21. Hogenboom A, Ketter W, van Dalen J, Kaymak U, Collins J, Gupta A. Adaptive tactical pricing in multi-agent supply chain markets using 
economic regimes. Decis Sci 2015;46:791-818.

22. Almeida RJ, Basturk N, Kaymak U. Probabilistic fuzzy systems for seasonality analysis and multiple horizon forecasts. In: Serguleva A, Almeida RJ, Palade V, Maringer D, editors. IEEE Conference on Computational Intelligence for Financial Engineering and Economics (CIFER)), London, UK: Piscataway: IEEE, March 27-28, 2014. p. 497-504.

23. Van Den Berg J, Kaymak U, Almeida RJ. Conditional density estimation using probabilistic fuzzy systems. IEEE Trans Fuzzy Syst 2013;21(5):869-82.

24. Tutmez B, Kaymak U, Tercan AE, Lloyd CD. Evaluating geoenvironmental variables using a clustering based areal model. Comput Geosci 2012;43:34-41.

25. Rai A. Application of artificial intelligence for virtually assisted prognosis of diabetes: A NODDS project. IJCA Proceedings on National Seminar on Application of Artificial Intelligence in Life Sciences 2013
NSAAILS. Vol. 1. February; 2013. p. 1-5.

26. Rai A, Ramanathan S. Distributed learning in networked controlled cyber physical system. Int J Pharm Technol 2016;8(3):18537-46.

27. Rai A. High performance computing: A reality at central-India. Int J Innov Res Dev 2013;2(3):734-43.

28. Rai A. Automation in computation over Linux integrated environment. J Adv Shell Program 2014;1(1):18-20.

29. Rai A. Air computing: A parallel computing module for offloading computational workload on neighboring android devices. Recent Trends Parallel Comput 2015;1(3):10-3.

30. Rai A. Parallelizing mutations for genetic algorithm. Recent Trends Parallel Comput 2015;3:7-9.

31. Rai A, Ramanathan S, Kannan RJ. Quasi opportunistic supercomputing for geospatial socially networked mobile devices. Enabling Technologies: Infrastructure for Collaborative Enterprises (WETICE), 2016 IEEE $25^{\text {th }}$ International Conference on. IEEE; 2016. 